\title{
Influência de circulações locais em áreas de aproveitamento de potencial eólico na região do Lago de Sobradinho
}

Influence of local circulations in areas of wind power development in the Sobradinho Lake region

\author{
Pollyanna Kelly de Oliveira Silva ${ }^{1}$, Maria Regina da Silva Aragão², Magaly de Fatima Correia ${ }^{2}$ e \\ Samira de Azevedo Santos ${ }^{1}$ \\ 1 Programa de Pós-Graduação em Meteorologia, UFCG, Campina Grande, PB, Brasil \\ pollyanna_kelly@yahoo.com.br; samiraazevedo22@gmail.com \\ ${ }^{2}$ Departamento de Ciências Atmosféricas, UFCG,Campina Grande,PB, Brasil \\ regina@dca.ufcg.edu.br; magaly@dca.ufcg.edu.br
}

\begin{abstract}
Resumo
Neste trabalho padrões de circulações locais são investigados para o ano de 2010 na região do Lago de Sobradinho, aos níveis de 25 e 50 metros. Os hodógrafos ilustram a variação do vento local nos quatro quadrantes da rosa dos ventos, na maioria dos meses. A hora local (HL) e magnitude das intensidades máximas e mínimas dependem da época do ano. Um cisalhamento vertical negativo, no vento local, durante a madrugadainício da manhã (noite) é mais evidente no período de maio a setembro (outubro a fevereiro). O vento local, nos horários de máxima intensidade, representa até $60 \%$ da velocidade do vento observado e, no restante das vinte e quatro horas do dia, entre 10 e 30\%. Essa razão decresce com a altura nos doze meses de 2010, e é mais evidente nos períodos de 4-6 HL e 17-20 HL, à exceção de março e junho, respectivamente. As circulações locais podem estar associadas ao acoplamento entre a brisa terrestre (lacustre) e os ventos catabáticos (anabáticos) durante a madrugada-início da manhã (final da tarde-início da noite). Os meses de junho, julho e agosto apresentam menos variabilidade na direção do vento, favorecendo um melhor aproveitamento das turbinas eólicas.
\end{abstract}

Palavras-chave: Sazonalidade. Brisa lacustre e terrestre. Vento anabático e catabático. Petrolina.

\begin{abstract}
In this work local circulation patterns are investigated for the year of 2010 in the Sobradinho Lake region, at the $25 \mathrm{~m}$ and $50 \mathrm{~m}$ levels. The hodographs illustrate the local wind variation in the four quadrants of the wind rose, in the majority of the months. The local time (LT) and magnitude of the maxima and minima intensities depend on the time of the year. A negative vertical shear in the local wind during the late night-early morning (night) is more evident in the period from May to September (October to February). The local wind, at times of maximum intensity, represents up to $60 \%$ of the observed wind speed and, in the remaining of the twenty four hours of the day, between 10 and 30\%. This ratio decreases with height in the twelve months of 2010, and is more evident in the periods 4-6 LT and 17-20 LT, with exception of March and June, respectively. The local circulations may be associated with coupling between the land (lake) breeze and the catabatic (anabatic) winds during the late nightearly morning (late afternoon-early night). The months of June, July and August show less variability in wind direction, favoring a better use of the wind turbines.
\end{abstract}

Keywords: Seasonality. Lake and land breeze. Anabatic and catabatic wind. Petrolina 


\section{Introdução}

Nos últimos anos investimentos para aproveitamento da energia gerada a partir dos ventos na região do Lago de Sobradinho tem crescido sistematicamente. Atualmente 33 usinas eólicas fazem parte da base de dados da Agência Nacional de Energia Elétrica (ANEEL) para essa região. Nela constam usinas que estão em operação, outorgadas, com Despacho de Requerimento de Outorga (DRO) vigente, e as cadastradas pela Empresa de Pesquisa Energética (EPE) para participarem dos leilões de comercialização de energia elétrica no Ambiente de Contratação Regulado (ACR). No total, a região perfaz um pouco mais de $1 \mathrm{GW}$ de potência instalada (www.sigel.aneel.gov.br).

A implantação do Lago de Sobradinho e o desenvolvimento regional decorrente de seus múltiplos usos estão na base desses investimentos. A importância do Lago sob o aspecto energético também se traduz no fato de que as circulações locais geradas devido a sua presença podem interagir com outras circulações locais e com a circulação de grande escala, impactando diretamente o potencial eólico regional.

Dessa forma, o objetivo deste trabalho é diagnosticar as circulações locais existentes no ano de 2010, e identificar e quantificar a sua possível contribuição para o aproveitamento da energia gerada a partir da força dos ventos na região do Lago de Sobradinho.

\section{2 Área de Estudo}

O estudo foi realizado para a região do Lago de Sobradinho, a partir de registros anemométricos da torre instalada em PetrolinaPE, disponibilizados pelo Projeto SONDA do INPE. A estação está situada a cerca de $60 \mathrm{~km}$ do Lago, 5 km do Rio São Francisco e 40 km de Petrolina. O relevo no entorno apresenta topografia acentuada a noroeste e a sudoeste da estação, com altitudes de até 550 metros e 640 metros, respectivamente (Figura 1).

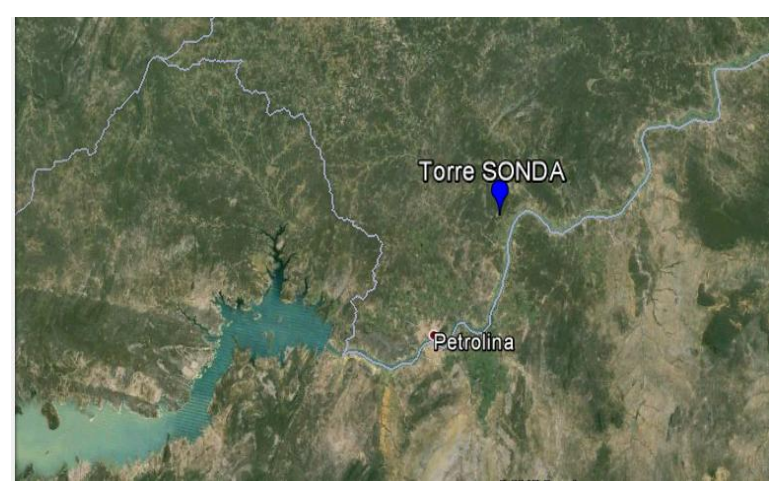

Figura 1 - Localização da estação anemométrica do projeto SONDA (Fonte: Google Earth Pro).

\section{Metodologia}

São utilizados dados de velocidade e direção do vento medido na altura de $25 \mathrm{~m}$ e $50 \mathrm{~m}$, com registros de 10 em 10 minutos para o ano de 2010.

Com o objetivo de minimizar os efeitos da circulação de grande escala e realçar a escala menor, o vento observado em cada horário foi subdividido em uma parte média diária, denominada vento de grande escala ou vento sinótico, e uma parte de desvio, o vento local horário. $\mathrm{Na}$ análise do vento local foram analisadas as médias horárias dos doze meses, obtendo assim a circulação local média de cada mês, para cada altura. A obtenção do vento local seguiu as seguintes etapas:

i) A componente zonal $(\mathrm{u})$ e meridional (v) do vento com base nas equações (1) e (2):

$$
\begin{aligned}
& u=-V \operatorname{sen}(\theta) \\
& v=-V \cos (\theta)
\end{aligned}
$$

ii) A média diária, ou vento de grande escala, utilizando as equações (3) e (4):

$$
\begin{aligned}
& \bar{u}=\frac{\sum_{k=1}^{24} u}{24} \\
& \bar{v}=\frac{\sum_{k=1}^{24} v}{24}
\end{aligned}
$$

iii) Os desvios horários para cada dia, ou vento local diário, com base nas equações (5) e (6):

$$
\begin{aligned}
& u^{\prime}=u-\bar{u} \\
& v^{\prime}=v-\bar{v}
\end{aligned}
$$


iv) A média mensal dos desvios horários, ou vento local médio mensal, usando (7) e (8):

$$
\begin{aligned}
& \overline{u^{\prime}}=\frac{\sum_{d=1}^{n} u}{n} \\
& \overline{v^{\prime}}=\frac{\sum_{d=1}^{n} v^{\prime}}{n}
\end{aligned}
$$

na qual n é o número de dias do mês.

A contribuição do vento (circulação) local com relação ao vento (circulação) observado foi avaliada para cada horário e mês através da razão entre suas velocidades médias mensais.

\section{Resultados e Discussões}

A variabilidade da circulação local nos níveis de 25 e 50 metros para o ano de 2010 é ilustrada por hodógrafos na Figura 2 e Figura 3, respectivamente, e também por sua intensidade na Figura 4. $\mathrm{O}$ horário e magnitude das intensidades máximas e mínimas dependem da época do ano (Figura 4). Na maioria dos meses, o vento local varia entre os quatro quadrantes da rosa dos ventos, nas vinte e quatro horas do dia (Figuras 2, 3). Dessa forma, ele pode inclusive atuar em direção oposta a do vento de grande escala. Esse é o caso, por exemplo, no horário de máxima intensidade do vento local no início da manhã, nos meses de junho, julho e agosto (Figura 2f-h, 3f-h, 4f-h), quando o vento observado (formado pela contribuição da grande escala e escala local) tem intensidade mínima (não mostrado).

As maiores intensidades da circulação local são registradas no mês de março, a $25 \mathrm{~m}$, e dezembro, nas duas alturas (Figuras 2c, 2l, 31). No caso das máximas, por exemplo, nos meses de julho e agosto o máximo principal ocorre às 6 $\mathrm{HL}$, e há dois secundários, às 9-10 HL e $19 \mathrm{HL}$ (Figuras 2g, 2h, 3g, 3h), enquanto que em fevereiro o máximo principal é visto às 19-20 HL, e os secundários às $5 \mathrm{HL}$ e $11 \mathrm{HL}$ (Figuras 2b, 3b). Dessa forma, máximos principais e secundários ocorrem predominantemente no início da manhã e final da tarde. Resultados de estudos anteriores (CORREIA e SILVA DIAS, 2003; FREITAS, 2003; CZARNOBI, 2006) sobre regiões influenciadas por lagos formados devido ao represamento para construção de hidrelétricas, evidenciam que as máximas intensidades da brisa lacustre (terrestre) ocorrem no final da tarde (início da manhã).

Outro aspecto a observar é que durante o primeiro e último trimestres do ano a rotação do vento local é predominantemente no sentido anti-horário (Figuras 2a-c, 3a-c, 2j-1, 3j-1). Estes resultados são similares àqueles obtidos em estudos precedentes para áreas com lagos (FREITAS, 2003; MELO et al., 2013).

A variação horária da razão percentual entre a velocidade do vento (circulação) local e a velocidade do vento (circulação) observado, e a variação horária da velocidade do vento local são ilustradas na Figura 4 para os níveis de 25 e 50 metros. Um cisalhamento vertical negativo, no vento local, durante a madrugada-início da manhã (noite) é mais evidente nos meses de maio a setembro (outubro a fevereiro) (Figuras $4 \mathrm{e}-\mathrm{i}, 4 \mathrm{a}, 4 \mathrm{~b}, 4 \mathrm{j}-\mathrm{l})$. Por outro lado, o vento observado tem cisalhamento vertical positivo (não mostrado). O vento local, nas horas de máxima intensidade, chega a representar até $60 \%$ da velocidade do vento observado (Figura 4f); para os demais períodos do dia os ventos locais representam entre 10 e 30\% (Figuras 4a-1). O decréscimo da razão com a altura é visto em todo o ano de 2010, com as máximas contribuições do vento local ocorrendo predominantemente entre 4-6 HL e 17-20 HL, exceto nos meses de março e junho, respectivamente (Figura 4). Esse padrão pode sugerir um acoplamento entre a brisa terrestre (lacustre) e os ventos catabáticos (anabáticos) no período da madrugada-início da manhã (final da tarde-início da noite).

No período de outubro a fevereiro os hodógrafos mostram ventos do quadrante noroeste (QNO) no final da tarde-noite e do quadrante sudoeste (QSO) na madrugada-início da manhã, enquanto que no período da manhã e tarde há variação entre os quadrantes nordeste (QNE) e sudeste (QSE) (Figuras 2a, 2b, 2j-1, 3a, $3 b, 3 j-1)$. Neste período a razão entre o vento local e o vento observado é maior à noite-início da manhã podendo indicar a presença dos ventos catabáticos, que escoam das áreas mais elevadas em direção à estação (Figuras 4a, 4b, 4j-1).

Os meses de junho, julho e agosto apresentam menos variabilidade na direção do vento local, se comparada aos demais meses do ano, já que no período da madrugada-início da manhã predominam ventos do QNO, manhã e tarde 

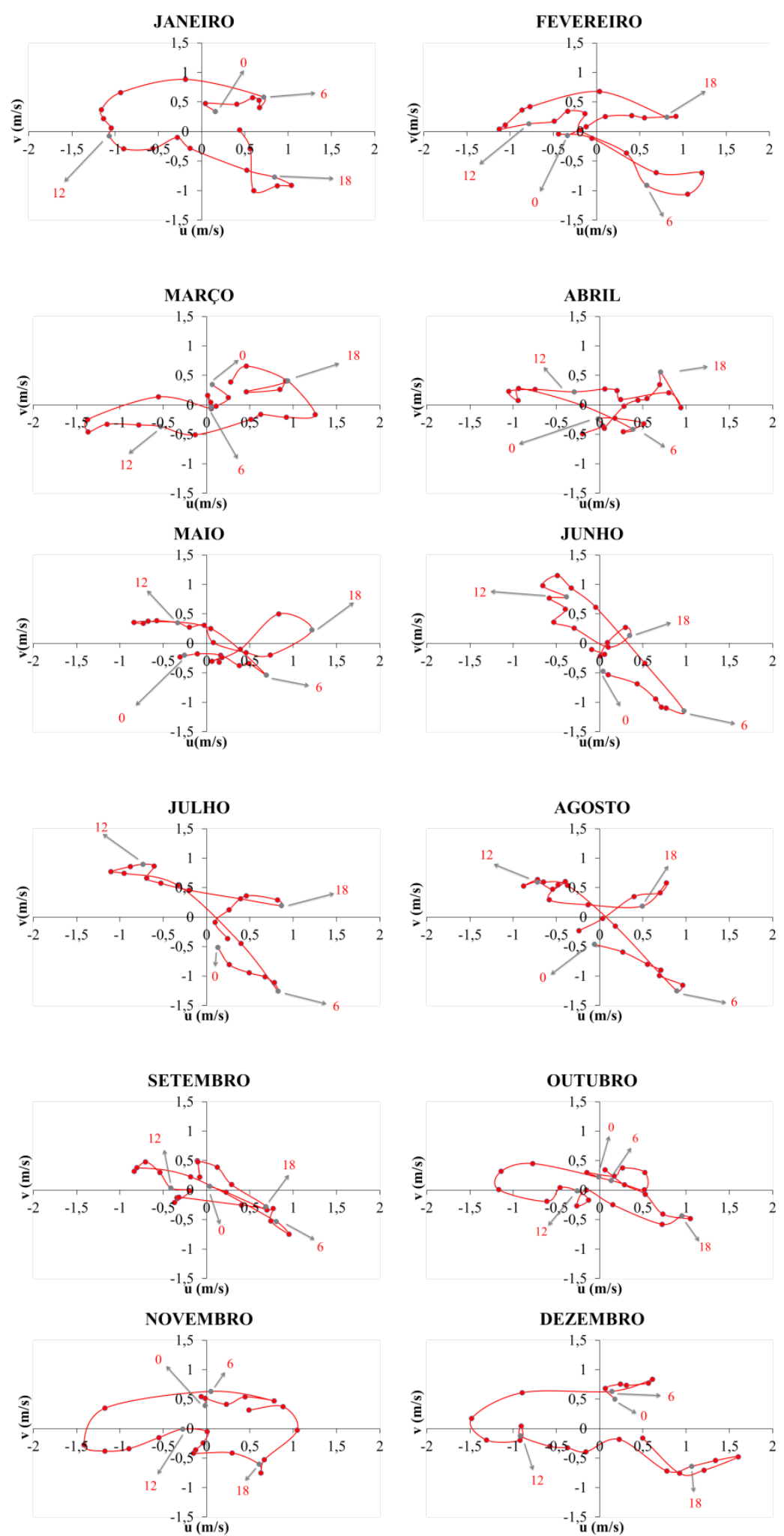

Figura 2 - Hodógrafos da circulação local média mensal (m/s) a $25 \mathrm{~m}$ para o período de (a) janeiro a (l) dezembro de 2010. Os números na cor vermelha assinalados por flechas representam a hora local em intervalos de 6 horas (Fonte dos dados: SONDA/INPE) 

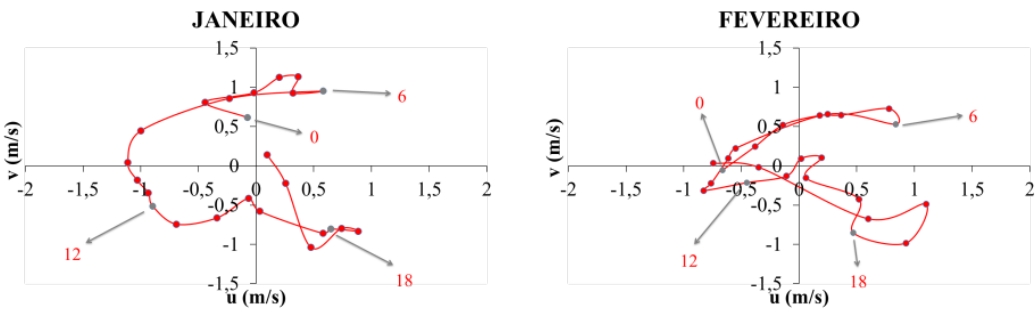

MARÇO
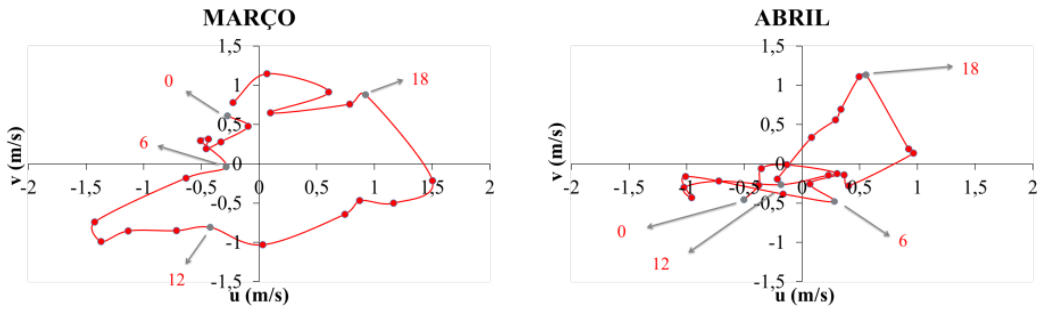

MAIO

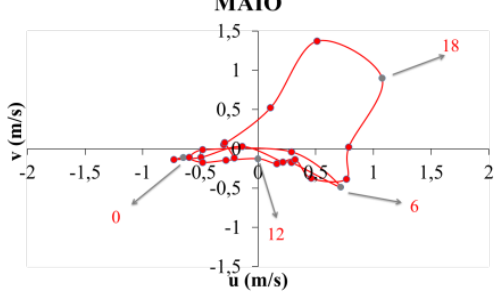

JUNHO

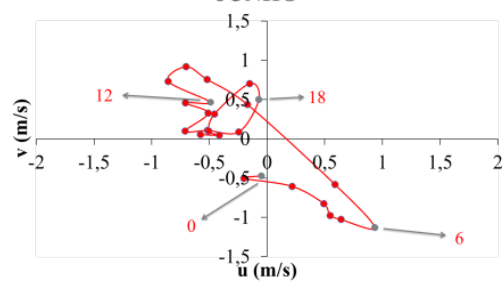

JULHO
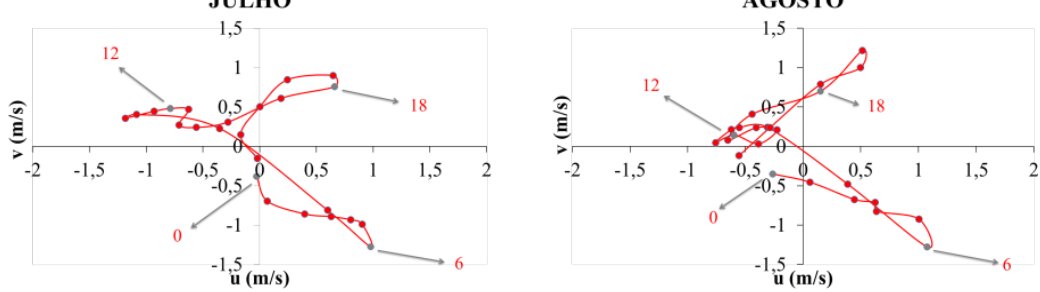

OUTUBRO

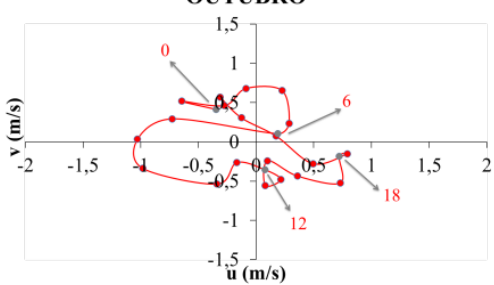

NOVEMBRO
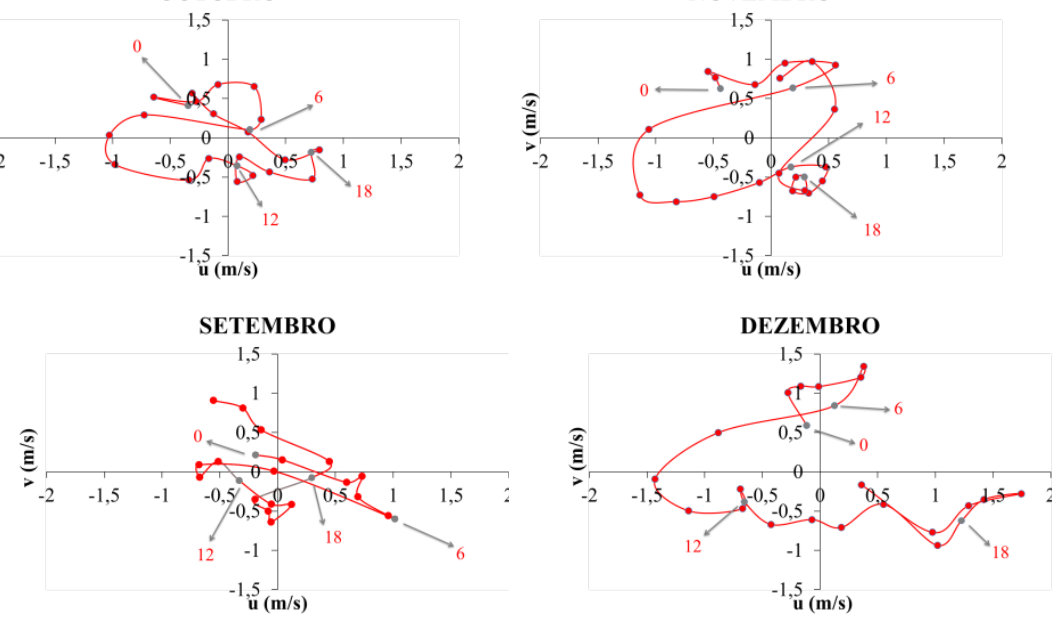

Figura 3 - Hodógrafos da circulação local média mensal (m/s) a $50 \mathrm{~m}$ para o período de (a) janeiro a (l) dezembro de 2010. Os números na cor vermelha assinalados por flechas representam a hora local em intervalos de 6 horas (Fonte dos dados: SONDA/INPE). 


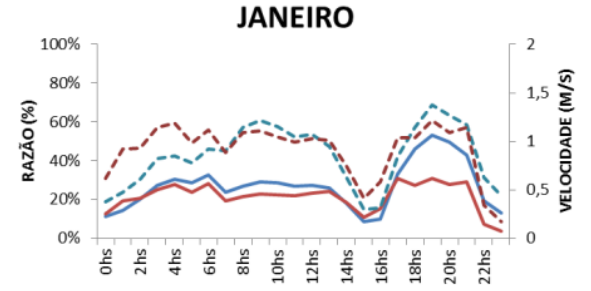

(a)

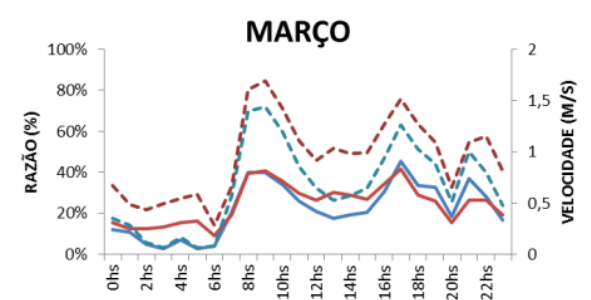

(c)

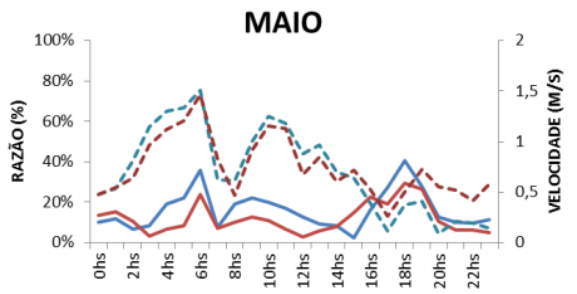

(e)

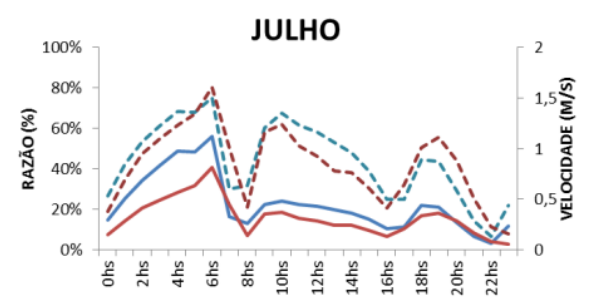

(g)

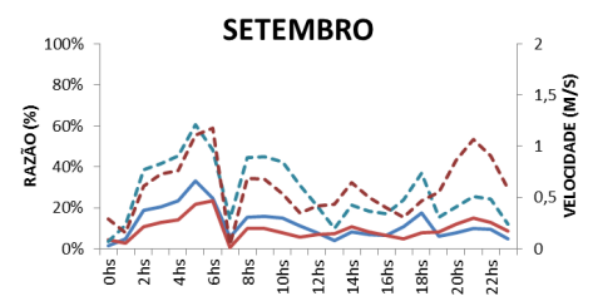

(i)

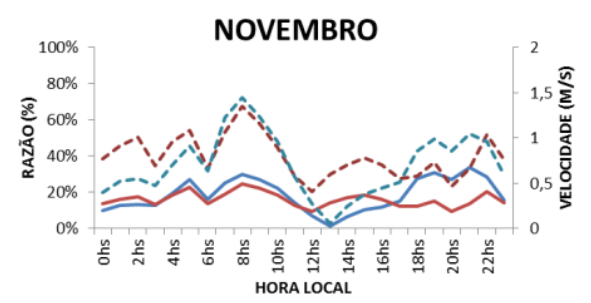

(k)

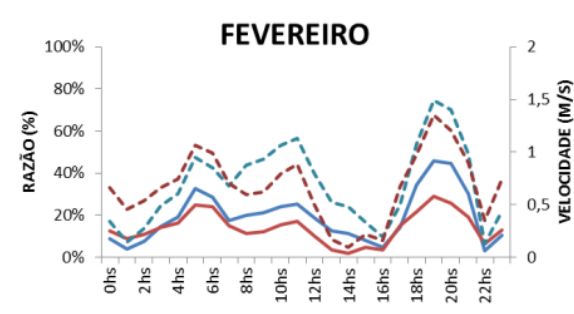

(b)

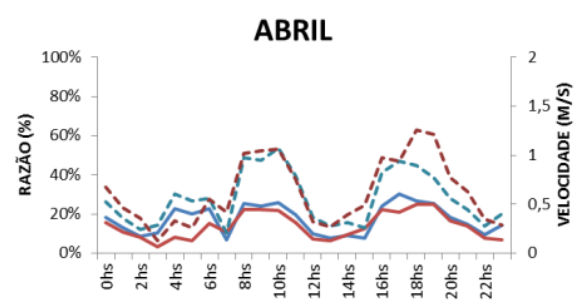

(d)

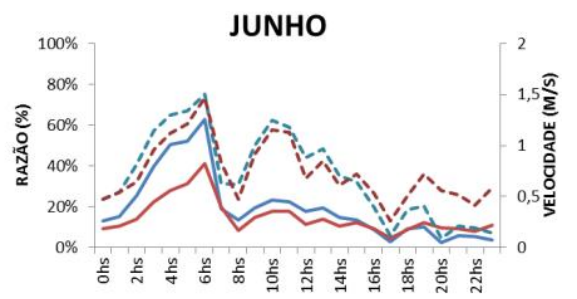

(f)

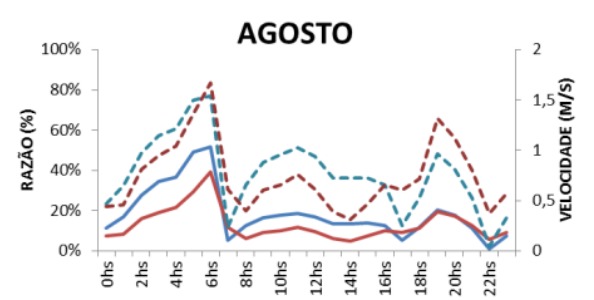

(h)

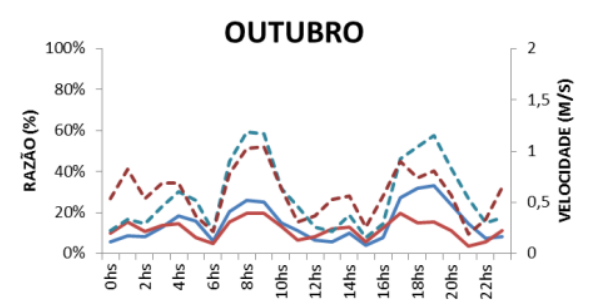

(j)

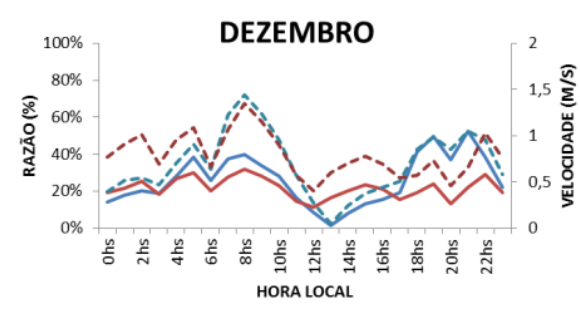

(1)

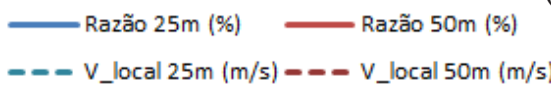

Figura 4 - Variação mensal da razão horária (\%) entre a velocidade do vento (circulação) local e a velocidade do vento observado, e da velocidade do vento (circulação) local (m/s), para os níveis de 25 m e $50 \mathrm{~m}$, no período de (a) janeiro a (l) dezembro de 2010 (Fonte dos dados: SONDA/INPE) 


\section{Referências}

CORREIA, M.F.; SILVA DIAS, M.A.F. (2003). Variação do Nível do Reservatório de Sobradinho e seu Impacto Sobre o Clima da Região. Revista Brasileira de Recursos Hídricos, v.8, n.1, p.157-168.

CLIMANÁLISE. (2010) Boletim de monitoramento e análise climática. Cachoeira Paulista, v.25, n. 1-12.

CZARNOBI, A.F.; PRUDÊNCIO, R.S.; RODRIGUES, M.L.G. (2006) A Circulação Atmosférica Local na Região da Usina de Itá. In:
Congresso Brasileiro de Meteorologia, XIV, 2006, Florianópolis. Anais... Florianópolis: CBMET.

FREITAS, E.D. (2003) Circulações locais em São Paulo e sua influência sobre a dispersão de poluentes. Tese (Doutorado em Ciências Atmosféricas) - Universidade de São Paulo, São Paulo.

MELO, E.C.S.; SILVA ARAGÃO, M.R.; CORREIA, M.F. (2013) Regimes do Vento à Superfície na Área de Petrolina, Submédio do Rio São Francisco. Revista Brasileira de Meteorologia, v.28, n.3, p. 229-241. 